\title{
Expanding the indications for the David V aortic root replacement: Early results
}

\author{
Bradley G. Leshnower, MD, ${ }^{\text {a }}$ Robert A. Guyton, MD, ${ }^{\text {a }}$ Richard J. Myung, MD, ${ }^{a}$ John D. Puskas, MD, ${ }^{a}$ \\ Patrick D. Kilgo, MSc, ${ }^{\mathrm{b}}$ LaRonica McPherson, RN, ${ }^{\mathrm{a}}$ and Edward P. Chen, $\mathrm{MD}^{\mathrm{a}}$
}

Objective: To examine the early results of the David V valve-sparing aortic root replacement procedure in expanded, higher risk clinical scenarios with appropriately selected patients.

\begin{abstract}
Methods: From 2005 to 2011, 150 David V valve-sparing aortic root replacements were performed within Emory Healthcare. A total of 78 patients (expanded group) had undergone the David V in expanded, difficult clinical settings such as emergent type A dissection $(n=29)$, grade $3+$ or greater aortic insufficiency (AI) $(n=53)$, or reoperative cardiac surgery $(n=14)$. These patients were evaluated and compared with a group of 72 patients (traditional group) with less than grade $3+\mathrm{AI}$ who underwent a David V in a traditional, elective setting. The mean follow-up was 19 months (range, 1-72), and the follow-up data were $88 \%$ complete.
\end{abstract}

Results: There were 3 operative deaths (2.2\%), all occurring in the expanded group. The overall patient survival at 6 years was $95 \%$. Three patients required aortic valve replacement: two for severe AI and one for fungal endocarditis. Both groups had concomitant cusp repairs performed in conjunction with the David V (traditional, $\mathrm{n}=10$; and expanded, $\mathrm{n}=16 ; P=.27$ ). At follow-up, freedom from moderate AI was $93 \%$, and the freedom from aortic valve replacement was $98 \%$. No significant difference was observed in the freedom from moderate AI between the expanded and traditional groups ( $91 \%$ vs $95 \%$, respectively; $P=.16)$.

Conclusions: In selected patients possessing appropriate aortic cusp anatomy, the David V can be safely and effectively performed for the expanded indications of aortic dissection, severe AI, and reoperative cardiac surgery with low operative risk. Valve function has remained excellent in the short term, providing evidence of durability and a low rate of valve-related complications. (J Thorac Cardiovasc Surg 2012;143:879-84)

Valve-sparing aortic root replacement has become the ideal operation for young patients with aortic root pathology and normal aortic valve cusps. Two techniques for this procedure were pioneered by David and Feindel ${ }^{1}$ (reimplantation) and Sarsam and Yacoub $^{2}$ (remodeling) and have been adopted worldwide by cardiac surgeons during the past 2 decades. Originally developed for the treatment of patients with Marfan syndrome with aortic root aneurysms and normal aortic cusps, the David reimplantation procedure has been modified since its initial description, ${ }^{3-8}$ and its use has gradually been expanded to other clinical scenarios. As the procedure has evolved, experts have expressed their concerns regarding the feasibility of performing a valve-sparing root replacement in patients with severe

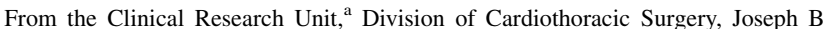
Whitehead Department of Surgery, Emory University School of Medicine, Atlanta, $\mathrm{Ga}$; and Rollins School of Public Health, ${ }^{\mathrm{b}}$ Emory University School of Medicine, Atlanta, Ga.

Disclosures: Authors have nothing to disclose with regard to commercial support.

Read at the 37th Annual Meeting of The Western Thoracic Surgical Association, Colorado Springs, Colorado, June 22-25, 2011.

Received for publication June 20, 2011; revisions received Dec 25, 2011; accepted for publication Jan 16, 2012; available ahead of print Feb 13, 2012.

Address for reprints: Edward P. Chen, MD, Division of Cardiothoracic Surgery, Emory Clinic, 1365 Clifton Road, Suite A2236, Atlanta, GA 30322 (E-mail: epchen@emory.edu).

$0022-5223 / \$ 36.00$

Copyright (c) 2012 by The American Association for Thoracic Surgery

doi:10.1016/j.jtcvs.2012.01.048 aortic insufficiency (AI) or in higher risk settings such as reoperative surgery or acute type A dissection. ${ }^{9-12}$

In a previous report, we described our experience of performing the David V reimplantation procedure (David V) in 37 patients in the setting of acute type A dissection, reoperative cardiac surgery, or severe AI. ${ }^{13}$ These patients had perioperative outcomes equivalent to those of a group of patients who had undergone a Bentall procedure in similar scenarios. The present report expands on our previous efforts by comparing the short-term results of a larger cohort of patients who underwent a David V in these higher risk scenarios with those of an elective cohort of patients with minimal AI who underwent a David V as their primary cardiac operation. The goal of the present analysis was to provide additional safety and durability data to evaluate the expanded use of the David V procedure. Broader application of this procedure for the treatment of complex root pathology in a wide variety of expanded, higher risk clinical settings will potentially enable more patients to avoid valve replacement and its inherent pitfalls.

\section{METHODS}

The present study was conducted with approval of the institutional review board of Emory University in compliance with the Health Insurance Portability and Accountability Act regulations and the Declaration of Helsinki. The institutional review board waived the need for individual patient consent. From January 2005 through June 2011, 150 patients 


\section{Abbreviation and Acronym \\ $\mathrm{AI}=$ aortic insufficiency}

underwent valve-sparing aortic root replacement using the David V reimplantation technique at Emory University-affiliated hospitals. These patients were divided into 2 groups according to the indications (traditional or expanded, higher risk clinical settings for which a valve-sparing root replacement procedure has been described). ${ }^{9-12}$ Of the 150 patients, 78 (expanded group) underwent a David V in the following situations: reoperative cardiac surgery $(\mathrm{n}=14)$, emergent acute type A aortic dissection $(n=29)$, or greater than moderate $\mathrm{AI}$ ( $\geq$ grade $3+; \mathrm{n}=53$ ). These patients were compared with a traditional, elective cohort of 72 patients (traditional group) who underwent a primary elective David V procedure for aortic root aneurysms with moderate AI or less $(\leq$ grade $2+$ ). All elective cases were evaluated with preoperative transesophageal echocardiography to evaluate the aortic root complex. The aortic valve cusps were carefully inspected for any signs of degeneration such as free margin thickening, calcification, and prolapse. The presence and degree of AI was noted, as well as the eccentricity of any regurgitant jets. The final decision on whether to proceed with a David V procedure was determined by direct inspection of the cusps intraoperatively after opening the root.

\section{Surgical Technique}

All procedures were performed through a median sternotomy using cardiopulmonary bypass. Patients undergoing isolated aortic root replacement received right atrial and central aortic cannulation. In patients requiring total proximal aortic replacement and arch reconstruction under hypothermic circulatory arrest, the right axillary artery was cannulated as previously described. ${ }^{14,15}$

Once the aorta was transected, the aortic cusps were carefully inspected for calcification, perforations, stress fenestrations, free margin elongation, and prolapse. After the decision was made to proceed with the David V, the root was circumferentially dissected down to the nadir of the aortic annulus. All abnormal sinus tissue was excised, leaving a 4- to 5-mm rim of aortic tissue along the annulus. Coronary buttons were dissected free and mobilized. The diameter of the aortic graft prosthesis was determined using a modification of the original David-Feindel formula: graft diameter $=\left[2 \times\left(\mathrm{H}_{\text {cusp }} \times 2 / 3\right)\right]+6-8 \mathrm{~mm} .{ }^{1}$ A ruler was used to measure the height of the cusp from the hinge point at the nadir of the annulus to the free margin edge at the nodule of Arantius. For both bicuspid and tricuspid valves, this manner of graft sizing allowed for a graft larger than the ideal sinotubular junction and annulus, which was subsequently plicated down at the top and bottom positions to create the neosinus segments.

All aortic prostheses used in the present series were woven polyester Gelweave (Vascutek; Terumo, Ann Arbor, Mich) grafts. Most prostheses were tailored straight tube grafts; however, the Gelweave Valsalva graft was used in certain cases, depending on surgeon experience and preference. In the cases with a tailored graft, the annulus was measured with a transparent valve sizer (Medtronic Freestyle; Medtronic, Minneapolis, Minn). If annular dilation was present, interrupted 3-0 polypropylene pleating sutures were used to plicate the annular end to the diameter of the valve sizer. Next, three 3-0 braided polyester horizontal mattress sutures were placed through the left ventricular outflow tract $2 \mathrm{~mm}$ below the nadir of the annulus and passed through the tailored end of the graft. Before seating, the commissural stitches were brought through the inside of the graft. The anchoring sutures were then tied to secure the graft to the annulus.

Next, the commissural sutures were pulled up vertically with outward radial traction, and the cusps were assessed for optimal coaptation. Once the ideal position of each commissure was determined, the sutures were passed through the graft and tied on the outside. The valve was then reimplanted into the graft using 3 running 4-0 polyprolene sutures starting at the nadir of each cusp. After valve reimplantation, the coronary buttons were reimplanted using 5-0 polypropylene sutures. The graft was then plicated between the tops of the commissures with interrupted 4-0 pleating stitches to create a neosinotubular junction and 3 neosinuses. The neoroot was inspected, and any necessary cusp repair procedures were performed at that time. Cusp repair procedures were performed with 6-0 polytetrafluoroethylene (Gore-Tex suture, WL Gore \& Associates, Flagstaff, Ariz) and included leaflet plication, free margin reinforcement, and commissural annuloplasty. When valve competence was satisfactory, the distal anastomosis was completed.

\section{Echocardiography}

Intraoperative transesophageal echocardiography was used for all patients. Transthoracic echocardiograms were obtained for $87 \%$ of the patients before discharge. The patients were followed up prospectively, and annual surveillance echocardiographic examinations were scheduled for all patients. The grade of AI was interpreted using color flow mapping and continuous wave Doppler echocardiography. AI was reported according to a semiquantitative scale as 0 (grade 0 ), trace (grade 0.5 ), mild (grade 1), moderate (grade 2), moderate-severe (grade 3), and severe (grade 4). The more severe grade of AI was recorded in cases in which the regurgitant jet was interpreted as in between 2 grades (eg, mild to moderate was recorded as moderate).

\section{Statistical Analysis}

The pre-, peri-, and postoperative variables were descriptively compared between the 2 risk groups (traditional and expanded). Bivariate analyses were performed using 2-sample $t$ tests and chi-square tests to determine whether the means and proportions, respectively, were different between groups. Kaplan-Meier analysis was performed to estimate the survival distributions across the risk groups. The product-limit estimate for 1-, 3-, and 6-year survival was calculated using the death dates for each patient provided by the Social Security Death Index.

\section{RESULTS}

The preoperative characteristics are listed in Table 1. The mean patient age was $45 \pm 13$ years and did not differ between the 2 groups. A significantly greater incidence of male gender, hypertension, and preoperative renal failure was present in the expanded group. The traditional group contained a greater percentage of patients with Marfan syndrome (traditional, 19\%; expanded, $6.5 \% ; P=.03$ ). The mean aortic root diameter was larger in the expanded group (expanded, $5.6 \mathrm{~cm}$; traditional, $5.2 \mathrm{~cm} ; P<.001$ ). Also, $65 \%$ of the expanded group had greater than moderate $\mathrm{AI}$ on the preoperative echocardiogram, including 35 patients $(45 \%)$ with severe AI. In contrast, $82 \%$ of the traditional group had less than moderate AI.

The cardiopulmonary bypass, crossclamp, and circulatory arrest times were significantly longer in the expanded group $(P<.05)$. This can be explained by the greater incidence of concomitant procedures performed in the expanded group. The additional procedures included hemiand total arch reconstruction under moderate hypothermic circulatory arrest, coronary artery bypass, and mitral valve repair procedures. Both cohorts contained patients who required cusp repairs in addition to the David V, with a slightly greater incidence in the expanded group (Table 2). 
TABLE 1. Preoperative characteristics

\begin{tabular}{|c|c|c|c|}
\hline Characteristic & $\begin{array}{c}\text { Traditional } \\
(\mathbf{n}=\mathbf{7 2})\end{array}$ & $\begin{array}{c}\text { Expanded } \\
(\mathrm{n}=\mathbf{7 8})\end{array}$ & $P$ value \\
\hline Age $(y)$ & $45.3 \pm 14$ & $45.8 \pm 12$ & .74 \\
\hline Male gender & $52(72)$ & $71(91)$ & $.01 *$ \\
\hline BMI $\left(\mathrm{kg} / \mathrm{m}^{2}\right)$ & $28 \pm 6$ & $29 \pm 6$ & .33 \\
\hline History of CVA & $2(3)$ & $4(5)$ & .48 \\
\hline NYHA class III/IV & $4(6)$ & $13(17)$ & .30 \\
\hline Diabetes mellitus & $8(11)$ & $3(4)$ & .16 \\
\hline Hypertension & $39(54)$ & $56(72)$ & $.04 *$ \\
\hline Renal failure & $2(3)$ & $16(21)$ & $<.01 *$ \\
\hline COPD & $5(7)$ & $11(14)$ & .14 \\
\hline Aortic root diameter $(\mathrm{cm})$ & $5.2 \pm 0.4$ & $5.6 \pm 0.8$ & $<.001^{*}$ \\
\hline Ejection fraction $(\%)$ & $59 \pm 7$ & $54 \pm 8$ & $<.001^{*}$ \\
\hline Bicuspid aortic valve & $13(18)$ & $10(13)$ & .17 \\
\hline Marfan syndrome & $14(19)$ & $5(6)$ & $.03^{*}$ \\
\hline Previous cardio-aortic surgery & 0 & $14(18)$ & $<.001 *$ \\
\hline Emergent type A dissection & 0 & $29(37)$ & $<.001 *$ \\
\hline Preoperative AI & & & $<.001^{*}$ \\
\hline Zero & $13(18)$ & $3(4)$ & \\
\hline Trace & $16(22)$ & $3(4)$ & \\
\hline Mild & $30(42)$ & $13(17)$ & \\
\hline Moderate & $13(18)$ & $8(10)$ & \\
\hline Moderate to severe & 0 & $16(21)$ & \\
\hline Severe & 0 & $35(45)$ & \\
\hline
\end{tabular}

Data presented as mean \pm standard deviation or numbers, with percentages in parentheses. BMI, Body mass index; CVA, cerebral vascular accident; NYHA, New York Heart Association; $C O P D$, chronic obstructive pulmonary disease; $A I$, aortic insufficiency. *Statistically significant.

The overall operative mortality for the present series was $2 \%$. There were 3 operative deaths, all in the expanded group. Two of the deaths occurred early in the series in patients who presented with acute type A aortic dissection and grade 4+ AI. One patient died of a massive perioperative stroke and 1 of right ventricular failure secondary to cocaine-induced cardiomyopathy. The third mortality was an elective case in a patient with

TABLE 2. Perioperative data

\begin{tabular}{lccc}
\hline \multicolumn{1}{c}{ Variable } & $\begin{array}{c}\text { Traditional } \\
(\mathbf{n = 7 2})\end{array}$ & $\begin{array}{c}\text { Expanded } \\
(\mathbf{n = 7 8})\end{array}$ & $\boldsymbol{P}$ value \\
\hline CPB & $223 \pm 44$ & $256 \pm 60$ & $<.01 *$ \\
Crossclamp & $198 \pm 39$ & $216 \pm 45$ & $.01 *$ \\
HCA & $26 \pm 13$ & $35 \pm 18$ & $.01 *$ \\
Hemiarch replacement & $33(46)$ & $42(54)$ & .21 \\
Total arch replacement & $5(7)$ & $8(10)$ & .44 \\
Cusp repairs & $10(14)$ & $16(21)$ & .27 \\
Mitral repairs & $3(4)$ & $4(5)$ & .60 \\
CABG & $4(6)$ & $12(15)$ & .17 \\
Ventilation time (hr) & $12.3 \pm 12$ & $42.3 \pm 101$ & $.01 *$ \\
ICU LOS (d) & $2.1 \pm 2$ & $3.3 \pm 5$ & $.04 *$ \\
Hospital LOS (d) & $6.7 \pm 4$ & $8.4 \pm 7$ & .07 \\
\hline
\end{tabular}

Data presented as mean \pm standard deviation or numbers, with percentages in parentheses. $C P B$, Cardiopulmonary bypass; $H C A$, hypothermic circulatory arrest; $C A B G$, coronary artery bypass grafting; $I C U$, intensive care unit; $L O S$, length of stay. ${ }^{*}$ Statistically significant. preoperative $3+\mathrm{AI}$ who died on postoperative day $3 \mathrm{sec}-$ ondary to acute liver failure. There were 4 other nonaortic-related late deaths, all in the expanded group. For the entire series, the survival at 6 years of follow-up was $95 \%$. Figure 1 shows the Kaplan-Meier survival curves for the expanded and traditional groups.

An additional 7 patients not included in the present analysis of 150 patients underwent a David V procedure but had excessive AI on release of the crossclamp. These 7 patients, who were in the first $20 \%$ of our cases, underwent conversion intraoperatively to a Bentall procedure (4 in the expanded group and 3 in the traditional group), with no mortality. Of the 150 patients, 9 required mediastinal reexploration for bleeding (expanded, 5; traditional, 4). Postoperative ventilator requirements were significantly longer in the expanded group (expanded, $42 \pm 101$ hours vs traditional, $12 \pm 12$ hours; $P=.01$ ). This contributed to longer intensive care unit and overall hospital lengths of stay for the expanded group (Table 2).

All patients left the operating room with less than grade $2+\mathrm{AI}$. Of the 2 groups, $61(84 \%)$ of the traditional and $67(89 \%)$ of the expanded group had annual surveillance follow-up echocardiograms. The mean length of follow-up was 19 months (range, 1-72). At data analysis, surveillance echocardiograms had been obtained for $29 \%$ of patients in the postoperative period alone, in $52 \%$ of patients at longer than 1 year, and in $26 \%$ of patients at longer than 3 years.

A total of 9 patients (traditional, 3; expanded, 6) who developed grade $2+$ or greater $\mathrm{AI}$ in the follow-up period. Of the 3 traditional patients, 1 developed fungal endocarditis after 13 months and required a homograft root, ascending, hemiarch replacement. The remaining 2 patients had grade $2+\mathrm{AI}$ on the echocardiogram at 3 and 6 years postoperatively. In the expanded group, 2 patients developed grade 4+ AI within 13 months of their original operation and

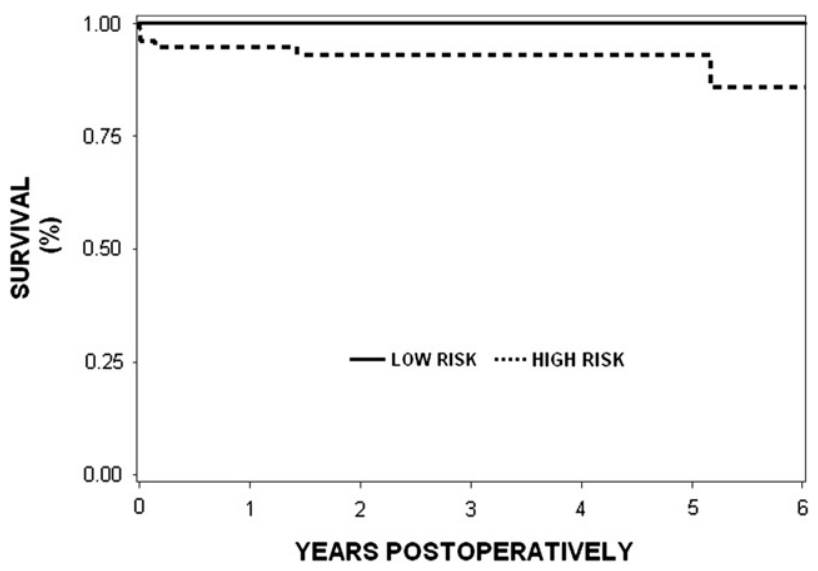

FIGURE 1. Survival of traditional and expanded patients after David V valve-sparing aortic root replacement. At follow-up, survival was $100 \%$ in the traditional group and $91 \%$ in the expanded group. 
underwent reoperative aortic valve replacement. Another 2 patients developed grade $3+\mathrm{AI}$ at 3 years of follow-up; 1 of these patients underwent a reoperative valve repair, which reduced the AI to grade $1+$, and the other was treated medically. Of the remaining 2 patients with grade $2+\mathrm{AI}$, 1 patient died of nonaortic-related causes, and 1 was lost to follow-up. The short-term freedom from moderate AI was $95 \%$ in the traditional group and $91 \%$ in the expanded group $(P=.16)$. The freedom from aortic valve-related reoperation was $99 \%$ in the traditional and $96 \%$ in the expanded groups (Figure 2). Overall, the freedom from aortic valve replacement for the series was $98 \%$.

\section{DISCUSSION}

After the initial report by David and Feindel ${ }^{1}$ describing the use of the reimplantation procedure in 10 patients, surgeons began to adopt the operation to treat patients with aortic root aneurysms and normal aortic valve cusps throughout the 1990s. In the past 10 years, surgeons have begun to modify the procedure and to use it in situations that were originally considered unconventional. In a previous study, we demonstrated the feasibility of performing a David V in certain higher risk subgroups with excellent short-term results. ${ }^{13}$ In the present report, we sought to provide more data to support expanding the indications for performing the David V in these higher risk scenarios.

In the present investigation, we reviewed the outcomes of 150 patients who underwent a David V reimplantation procedure. To evaluate the safety and efficacy of the David V in the higher risk population, we used our conventional David $\mathrm{V}$ cohort of patients as internal controls. Both groups were composed of young patients with a mean age of 45 years. The traditional group had a significantly larger percentage of patients with Marfan syndrome (traditional, 19\%; expanded, $6 \% ; P=.03$ ). Most of these patients had root aneurysms, minimal AI, and normal cusps and were ideal patients for a David V. The expanded cohort contained a more complex group of patients with a greater incidence

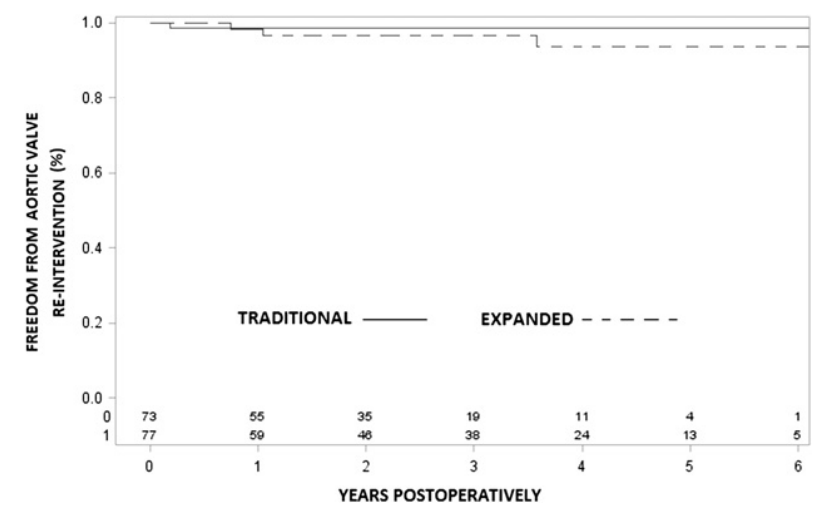

FIGURE 2. Freedom from aortic valve-related reoperation after David V valve-sparing aortic root replacement. At follow-up, freedom from reintervention was $99 \%$ in the traditional group and $96 \%$ in the expanded group. of preoperative renal failure. These patients required more concomitant procedures, in addition to the David V, and had longer cardiopulmonary bypass, crossclamp, and circulatory arrest times. These factors undoubtedly contributed to their longer ventilator requirements and intensive care unit and hospital lengths of stay.

As specified by the design of the study, the patients in the expanded group included all those with greater than moderate AI preoperatively. Severe AI has been considered by some surgeons to be a relative contraindication to a valvesparing procedure, especially if it is chronic AI with an eccentric jet. ${ }^{12,16}$ Severe AI is not a risk factor for operative death, but concern exists that in the setting of long-standing severe AI, the cusps could be intrinsically damaged, causing sclerosis, elongation of the free margin, and prolapse. These cases often require repair to 1 or more cusps, in addition to root reconstruction, and, therefore, carry the correspondent risk of failure in both the short and long term. ${ }^{12}$ Because of these concerns, patients with severe AI were placed in the expanded group.

In a recent series examining the results of valve-sparing procedures (remodeling or reimplantation) performed in the setting of severe AI, 5 of 6 patients who required subsequent aortic valve replacement had severe AI preoperatively. The freedom from greater than mild AI at 5 years was $88 \%$ in patients with less than severe preoperative AI compared with $50 \%$ in patients with severe preoperative AI. ${ }^{17}$ In our series, 35 patients $(23 \%)$ had severe AI preoperatively, 7 of whom had bicuspid valves and 12, type A dissection. In this subgroup, a single patient with a trileaflet aortic valve developed recurrent severe AI requiring valve replacement. Freedom from moderate AI was $100 \%$ for the remaining 34 patients. Excellent long-term valve function has been documented by others who have performed David Vs in the setting of preoperative severe AI. ${ }^{18,19}$

Because of its technical complexity and reliance on proper 3-dimensional reconstruction of the aortic root, the David V reimplantation procedure has been described as unforgiving with respect to small technical errors. ${ }^{7}$ This has given caution to many surgeons who have entertained the idea of performing a valve-sparing procedure in the setting of emergent repair of an acute type A aortic dissection. ${ }^{22}$ The surgical treatment of acute type A aortic dissection still carries a $24 \%$ in-hospital mortality rate according to the most recent International Registry of Acute Aortic Dissection report. ${ }^{20}$ However, patients presenting with type A dissection are often young with normal cusps. If the dissection involves the root, or the patient has a concomitant root aneurysm, valve-sparing root replacement might be an ideal operation. In the present study, 29 patients underwent a David V procedure in the setting of emergent type A repair. All 29 patients underwent arch reconstruction, with 3 patients undergoing total arch replacement. There were 2 operative deaths, for a mortality rate of 
$6.9 \%(2 / 29)$ in this subgroup. On follow-up echocardiography, valve function was preserved, with a $93 \%$ freedom from moderate AI. These data add to the work of others who have reported excellent long-term valve function and low mortality with valve-sparing procedures in the setting of acute type A aortic dissection. ${ }^{18,21,22}$ It must be emphasized that survival remains a primary goal in the surgical treatment of type A aortic dissection. Therefore, we share the opinions of others who have recommended that the reimplantation procedure should be performed in the setting of acute type A dissection only after gaining sufficient experience with the operation in the elective setting. $^{21}$

The third subgroup addressed in the present study was patients undergoing reoperative cardiac surgery. The David V reimplantation technique requires an extensive circumferential dissection of the aortic root down to a level below the nadir of each sinus. Only by reaching this level can the aortic annulus be appropriately anchored inside the graft (preventing future annular dilation), which represents a fundamental step of the procedure. In the reoperative setting, circumferential dissection of the root down to the level of the nadir of the sinus segments can be challenging, depending on the severity of the adhesions. The pulmonary artery, right ventricular outflow tract, right atrium, and left atrium can all be tightly adhered to the sinuses and are at risk of inadvertent injury that can go unrecognized until crossclamp removal. Because of these concerns, a preference for performing the remodeling procedure in lieu of reimplantation in the reoperative setting has been expressed. ${ }^{10}$ In our series, 14 patients $(18 \%)$ in the expanded group were undergoing reoperation. Of these 14 cases, 8 were previous aortic cases, including 5 previous type A dissection repairs. Two patients presented with a previous Ross procedure with dilated autografts. In both of these patients, the aneurysmal sinus tissue was excised, and the valve was successfully reimplanted inside a tailored graft. In this subgroup of 14 patients, 1 patient developed severe AI and required aortic valve replacement and 2 patients developed mild AI. The remainder had 0 or trace AI during follow-up.

Both groups contained patients who underwent cusp repair as a part of their reimplantation procedure. Although originally developed for patients with normal cusps, the expanded use of valve-sparing procedures during the past decade has largely been driven by the ability of surgeons to repair imperfect, prolapsed cusps with techniques such as cusp plication, free margin reinforcement, and triangular resection. ${ }^{16,23,24}$ Many of the large valve-sparing series reported contain a significant number of patients who required cusp repairs. ${ }^{18,23,25}$ However, there have been reports that the need for cusp repair is associated with the development of postoperative AI and reoperation for valve replacement. ${ }^{25,26}$ This raises the inevitable question of whether these patients should have undergone a Bentall procedure as their primary operation. In the present series, we performed cusp repairs in 26 patients $(17 \%$; traditional, 10; expanded, 16), with acceptable outcomes. There was 1 death and 1 patient who required subsequent aortic valve replacement. The freedom from moderate AI in the remaining patients was $92 \%$. This cohort of patients will be closely monitored for the development of significant $\mathrm{AI}$ and the need for aortic valve reintervention.

The limitations of the present study included the inherent selection bias from the study design and ongoing follow-up. Valve-sparing procedures are complex operations that were originally designed for a limited number of patients with aortic root aneurysms and normal aortic valve cusps. In attempting to expand the indications in which the David $\mathrm{V}$ reimplantation procedure can be performed, we were subject to inherent selection bias when offering this operation to patients, especially those in the expanded group. During the period covered by the present retrospective review, the patients in the expanded group represented approximately $10 \%$ of all our reoperative aortic procedures, $10 \%$ of all aortic dissections, and $15 \%$ of all our aortic root replacements. These patients were carefully selected to be a candidate for the David V only if their aortic cusp anatomy was believed to be appropriate for successful and durable repair. We captured follow-up echocardiograms for $88 \%$ of the patients in the present study. Follow-up echocardiographic surveillance is continuous and ongoing, and fewer cases were done earlier in the study period. At data analysis, a significant percentage $(29 \%)$ of these studies had only been obtained in the early postoperative period. Most patients had follow-up for longer than 12 months, and $26 \%$ have had follow-up for longer than 3 years. In addition, data regarding left ventricular size was not collected as a part of the present report. Ongoing prospective assessment of valve function continues, and additional durability data are being gathered. Owing to the follow-up period in the present study, the longer term success of the valve repairs has not been as thoroughly assessed as the shortterm valve function.

In conclusion, the data presented in the present report support the expanded use of the David V valve reimplantation procedure for aortic root reconstruction in the setting of emergent type A dissection, reoperative cardiac surgery, and severe AI in experienced hands. No significant differences were found when comparing the outcomes between the traditional and expanded groups. Operative mortality was low, and valve function in early follow-up remained durable. Valve-sparing aortic root replacement is an optimal therapy for aortic root pathology in suitable patients. It offers the patient the potential prospect of definitive treatment while avoiding the lifelong burden of anticoagulation or the inevitability of a second valve operation. The followup data reported in the present study were relatively short compared with the valve function for commercially 
available prosthetic aortic valves. Therefore, long-term surveillance is mandatory and will ultimately determine whether patients undergoing these procedures in these expanded clinical settings are truly receiving the optimal therapy.

\section{References}

1. David TE, Feindel CM. An aortic valve-sparing operation for patients with aortic incompetence and aneurysm of the ascending aorta. J Thorac Cardiovasc Surg. 1992;103:617-22.

2. Sarsam MA, Yacoub M. Remodeling of the aortic valve annulus. J Thorac Cardiovasc Surg. 1993;105:435-8.

3. David TE. The aortic valve-sparing operation. J Thorac Cardiovasc Surg. 2011; 141:613-5.

4. David TE, Armstrong S, Maganti M, et al. Long-term results of aortic valve sparing operations in patients with Marfan syndrome. J Thorac Cardiovasc Surg. 2009;138:859-64.

5. Gleason TG. New graft formulation and modification of the David reimplantation technique. J Thorac Cardiovasc Surg. 2005;130:601-3.

6. Patel ND, Williams JA, Barreiro CJ, Bethea BT, Fitton TP, Dietz HC, et al. Valvesparing aortic root replacement: early experience with the De Paulis Valsalva graft in 51 patients. Ann Thorac Surg. 2006;82:548-53.

7. Miller DC. Valve-sparing aortic root replacement in patients with the Marfan syndrome. J Thorac Cardiovasc Surg. 2003;125:773-8.

8. Svensson LG, Cooper M, Batizy LH, Nowicki ER. Simplified David reimplantation with reduction of annular size and creation of artificial sinuses. Ann Thorac Surg. 2010;89:1443-7.

9. De Oliveira NC, David TE, Ivanov J, Armstrong S, Eriksson MJ, Rakowski H, et al. Results of surgery for aortic root aneurysm in patients with Marfan syndrome. J Thorac Cardiovasc Surg. 2003;125:789-96.

10. Griepp RB, Lytle BW, David TE, Schafers HJ, Kallenbach K, Galla JD, et al. Session 1-ascending aorta from the Aortic Surgery Symposium VIII. Ann Thorac Surg. 2002;74:S1792-9.

11. Leyh RG, Fischer S, Kallenbach K, Kofidis T, Pethig K, Harringer W, et al. High failure rate after valve-sparing aortic root replacement using the "remodeling technique" in acute type A aortic dissection. Circulation. 2002;106(Suppl 1): I229-33.

12. De Paulis R. Aortic root surgery: from valve sparing to "spare and plasty." Eur J Cardiothorac Surg. 2010;38:513-4.
13. Kerendi F, Guyton RA, Vega JD, Kilgo PD, Chen EP. Early results of valvesparing aortic root replacement in high-risk clinical scenarios. Ann Thorac Surg. 2010;89:471-8.

14. Leshnower BG, Myung RJ, Kilgo PD, Vassiliades TA, Vega JD, Thourani VH, et al. Moderate hypothermia an unilateral selective antegrade cerebral perfusion: a contemporary cerebral protection strategy for aortic arch surgery. Ann Thorac Surg. 2010;90:547-54.

15. Halkos ME, Kerendi F, Myung R, Kilgo P, Pusaks JD, Chen EP. Selective antegrade cerebral perfusion via right axillary artery cannulation reduced morbidity and mortality after proximal aortic surgery. J Thorac Cardovasc Surg. 2009;138:1081-9.

16. Miller DC, Bachet J, Cameron DE, Cohen O, David TE, El Khoury G. Session I-ascending aorta from the Aortic Surgery Symposium X. Ann Thorac Surg. 2007;83:S785-90.

17. Baidu CC, Eichinger W, Bleiziffer S, Hermes G, Hettich I, Krane M, et al. Should root replacement with aortic valve-sparing be offered to patients with bicuspid valves or severe aortic regurgitation? Eur J Cardiothorac Surg. 2010;38:515-22.

18. David TE, Maganti M, Armstrong S. Aortic root aneurysm: principles of repair and long-term follow-up. J Thorac Cardiovasc Surg. 2010;140:S14-9.

19. Kallenbach K, Karck M, Leyh RG, Hagl C, Walles T, Harringer W, Haverich A. Valve-sparing aortic root reconstruction in patients with significant aortic insufficiency. Ann Thorac Surg. 2002;74:S1765-8, S1792-A1799.

20. Trimarchi S, Eagle KA, Nienaber CA, Rampoldi V, Jonker FH, De Vincentiis C, et al. Role of age in acute type A aortic dissection outcome: report from the International Registry of Acute Aortic Dissection (IRAD). J Thorac Cardiovasc Surg. 2010;140:784-9.

21. Kallenbach K, Karck M, Pak D, Salcher R, Khaladj N, Leyh R, et al. Decade of aortic valve sparing reimplantation: are we pushing the limits too far? Circulation. 2005;112:I-253-9.

22. Erasmi AW, Stierle U, Bechtel JF, Schmidtke C, Sievers HH, Kraatz EG. Up to 7 years' experience with valve-sparing aortic root remodeling/reimplantation for acute type A dissection. Ann Thorac Surg. 2003;76:99-104.

23. Miller DC. Valve-sparing aortic root replacement: current state of the art and where are we headed? Ann Thorac Surg. 2007;83:S736-9.

24. Gleason TG. Current perspective on aortic valve repair and valve-sparing aortic root replacement. Semin Thorac Cardiovasc Surg. 2006;18:154-64.

25. Hanke T, Charitos EI, Stierle U, Robinson D, Gorski A, Sievers HH, et al. Factors associated with the development of aortic valve regurgitation over time after two different techniques of valve-sparing aortic root surgery. $J$ Thorac Cardiovasc Surg. 2009; 137:314-9.

26. De Paullis R, Scaffa R, Nardella S, Maselli D, Weltert L, Bertoldo F, et al. Use of the Valsalva graft and long-term follow up. J Thorac Cardiovasc Surg. 2010;140:S23-7. 